\title{
QUALITATIVE BEHAVIOR OF CONSERVATION LAWS WITH REACTION TERM AND NONCONVEX FLUX
}

\author{
BY \\ CORRADO MASCIA \\ Dipartimento di Matematica “G. Castelnuovo", Università degli Studi di Roma "La Sapienza", P.le \\ Aldo Moro 5, I-00185 Roma, Italy
}

Abstract. The aim of the paper is to study qualitative behavior of solutions to the equation

$$
\frac{\partial u}{\partial t}+\frac{\partial f(u)}{\partial x}=g(u)
$$

where $(x, t) \in \mathbb{R} \times \mathbb{R}_{+}, u=u(x, t) \in \mathbb{R}$. The main new feature with respect to previous works is that the flux function $f$ may have finitely many inflection points, intervals in which it is affine, and corner points. The function $g$ is supposed to be zero at 0 and 1 , and positive in between.

We prove existence of heteroclinic travelling waves connecting the two constant states for opportune choice of speeds. Finally, we analyze the large-time behavior of the Riemann problem with values 0 and 1 , showing convergence to one of the travelling waves. The speed of the limiting profile is explicitly characterized.

1. Introduction. Recently many results have been proved on the qualitative behavior of the scalar balance law

$$
\frac{\partial u}{\partial t}+\frac{\partial f(u)}{\partial x}=g(u)
$$

where $(x, t) \in \mathbb{R} \times \mathbb{R}_{+}, u=u(x, t) \in \mathbb{R}([1]-[3],[6],[7],[13]-[19],[21]-[24])$. These aim at explaining the new asymptotic patterns appearing when considering (1.1) as a conservation law with an additional source term. Towards that end, the source term $g$ was allowed to be quite general whereas the flux term $f$ was constrained to be regular and strictly convex. At most $f$ has been allowed to have one or two inflection points ([4], [15], [23]). The convexity hypothesis is strong. Indeed it implies an ordering property on propagation speeds of characteristic curves, due to the fact that $f^{\prime}$ is increasing. In [16], extending a result proved in [23], it is explicitly shown that this hypothesis is too restrictive to understand the behavior in the general case. In fact it is proved,

Received September 9, 1998.

2000 Mathematics Subject Classification. Primary 35L60; Secondary 74J30.

Key words and phrases. Balance laws, travelling waves, asymptotic behavior, Riemann problem.

E-mail address: mascia@mat.uniroma1.it 
under assumptions on $f$ and $g$, that the solution of a Cauchy problem for (1.1) with monotone initial datum is discontinuous until some finite time, and thereafter becomes continuous; on the contrary, in [1] it has been proved that in the regular convex case, when a discontinuity comes into view, it cannot disappear. This leads us to expect different behavior in the qualitative analysis of (1.1) under weaker convexity assumptions.

The aim of the present paper is to allow more freedom in the choice of the function $f$. In particular, under our hypotheses, the flux $f$ may have the following properties: finitely many inflection points, intervals in which it is an affine function, and corner points. Therefore, the class of possible fluxes is widely enlarged with respect to previous results. Precise assumptions on $f$ will be given later (see Sec. 2).

The main tool in the convex case is the technique of generalized characteristics ([1]-[2]). When $f$ has one inflection point the technique can still be adapted, but it becomes quite complicated ([15]). In the case of two (or more) changes of convexity, many properties of generalized characteristic do not hold, and this tool does not give information on qualitative behavior.

On the contrary, our approach applies to more general cases. It amounts to constructing the expected large-time profile of the solution (looking for entropy travelling waves of the problem) and then, by repeated use of the comparison principle for entropy solutions, to proving the convergence to the given profile.

We suppose that the function $g$ has two zeroes, and is positive in between. The role of the term $g$ can easily be understood by considering solutions of (1.1) independent of the space variable $x$. The evolution is governed by the ordinary differential equation

$$
\frac{d U}{d t}=g(U)
$$

Every $s \in \mathbb{R}$, such that $g(s)=0$, is a constant solution of (1.1). The stability character of such solutions (with respect to Eq. (1.2)) depends on the sign of $g$ near $s$. In our case the smaller zero is unstable, while the other is stable.

The assumption on the sign of the function $g$ is not essential for our analysis. On the contrary, the hypothesis on the number of zeroes of $g$ is important. Such choice is suggested by the analysis of balance laws with convex flux (see [18]). Indeed, under the convexity assumption, it can be proved that the asymptotic behavior of the solution to the Riemann problem is given by a pattern composed of some elementary waves. In the case of two zeroes, the pattern is made of a single wave. Therefore, our choice of $g$ should (and does) give rise to a solution converging to a single wave as $t \rightarrow+\infty$.

Qualitative analysis of $\mathrm{Fq}$. (1.1) gives information on the evolution of solutions when the reactive phenomenon, sketched above, is coupled with the convective mechanism of transport of the quantity $u$. represented by the flux function $f$. We will refer to these kinds of equations as reaction-convection ( $\mathrm{RC})$.

Similar studies have been made for a long time for reaction-diffusion equations (RD), both for the scalar case and for systems (see [26] and references therein). On the contrary, to the author's knowledge, the qualitative analysis of hyperbolic systems with a nondissipative reaction term is still at the starting point.

In the scalar case, RD and RC equations have some features in common. Both problems exhibit the same property concerning heteroclinic travelling waves: once fixing the 
asymptotic states of the wave, there is a limiting speed, say $c_{0}$, such that travelling fronts exist if and only if the speed belongs either to $\left(-\infty, c_{0}\right]$, or $\left[c_{0},+\infty\right)$, depending on the asymptotic states.

In [8] it was proved that the solution to a Riemann Problem for an RD equation converges (in a certain sense) to a travelling profile with limiting speed. In this work we prove an analogous result (Theorem 2.4) for $\mathrm{RC}$ equations.

Note that similar results on existence of travelling waves can be proved in the case of the simultaneous presence of reaction, diffusion and convection (RDC equations). It would be interesting, both from the point of view of mathematics and applications, to give a more complete analysis for this problem, for example, concerning the large-time behavior of solutions. Some new results on the subject are contained in [5].

Now we draw a sketch of the new results contained in this work. The precise statements will be given in Sec. 2 .

A typical question to be answered is the following. Let us consider an initial datum for the Cauchy problem for (1.1) of Riemann type, with the two constant values given by the two constant solutions of (1.1). How do these two different values of $u$ interact? That is: which is the behavior of the solution as time increases?

Following the results in the convex case, we look for the possible asymptotic profiles as heteroclinic travelling wave solutions of (1.1). We prove that there exists an extremal speed such that this kind of front exists if and only if the speed is either greater than or smaller than this extremal value. Moreover, we exhibit the existence of such waves in a constructive way. This permits us to have precise results on regularity and on the structure of the jump set of the profiles.

Then we turn our attention to the large-time behavior. We prove, by the construction of an appropriate family of entropy super- and subsolutions of (1.1), the convergence of the solution of the Riemann problem to the travelling wave with extremal speed.

The plan of the paper is as follows.

In Sec. 2, we give the precise assumptions on the functions $f$ and $g$ and the statement of the main results.

Section 3 is concerned with entropy travelling waves for (1.1). In order to prove uniqueness of such profiles with a given speed, we need some results on entropy solution of ordinary differential equations. Then we prove the existence for an appropriate choice of speed.

The content of Sec. 4 is the proof of the result on asymptotic behavior of solutions. We construct some useful supersolution by joining together different entropy solutions of (1.1). Results on large-time profiles are consequences of comparison principles.

2. Mathematical framework and results. We consider the Cauchy problem

$$
\begin{cases}\frac{\partial u}{\partial t}+\frac{\partial f(u)}{\partial x}=g(u), & (x, t) \in \mathbb{R} \times \mathbb{R}_{+}, \\ u(x, 0)=u_{0}(x), & x \in \mathbb{R} .\end{cases}
$$

Here $u$ is a real-valued function of the variables $(x, t)$. 
The initial data $u_{0}$ will be chosen with range in $[0,1]$; therefore, by the comparison principle, the solutions to (2.1) will have the same property. This allows us to impose assumptions on $f$ and $g$ just in the interval $[0,1]$.

We make the following hypotheses on the functions $f$ and $g$ :

$$
\left\{\begin{array}{l}
f \in C([0,1]), \quad f(0)=f^{\prime}(0)=0 \\
\exists 0 \equiv a_{0}<a_{1}<\cdots<a_{n+1} \equiv 1 \text { such that } f \in C^{1}\left(\left[a_{i}, a_{i+1}\right]\right) \text { and } \\
f^{\prime} \text { is either strictly monotone or constant in any interval }\left(a_{i}, a_{i+1}\right)
\end{array}\right.
$$

$$
g \in \operatorname{Lip}([0,1]), \quad g(0)=g(1)=0, \quad g(s)>0 \quad \forall s \in(0,1) .
$$

Note that no assumption has been made on the multiplicity of 0 and 1 as zeroes of $g$. Moreover, the hypotheses $f(0)=f^{\prime}(0)=0$ are not restrictive.

We denote with $f_{+}^{\prime}(s)$ and $f_{-}^{\prime}(s)$ the right, respectively, the left derivative of $f$ at $s$.

It is well known (see [9]) that under assumptions $(\mathrm{F})$ and $(\mathrm{G})$ the Cauchy problem has a unique entropy solution, i.e., for any positive $\psi \in C_{0}^{\infty}\left(\mathbb{R} \times \mathbb{R}_{+}\right)$,

$$
\begin{aligned}
\iint_{\mathbb{R} \times \mathbb{R}_{+}}\left[|u-k| \frac{\partial \psi}{\partial t}\right. & +\operatorname{sgn}(u-k)(f(u)-f(k)) \frac{\partial \psi}{\partial x} \\
& +\operatorname{sgn}(u-k) g(u) \psi] d x d t \geq 0 \quad \forall k \in \mathbb{R}
\end{aligned}
$$

and

$$
\text { ess } \lim _{t \rightarrow()^{+}} \int_{a}^{b}\left|u(x, t)-u_{0}(x)\right| d x, \quad-\infty<a<b+\infty .
$$

In the case of piecewise smooth solutions, formula (2.2) may be replaced by an easier criterion. Let $N \in \mathbb{N}$ and $\Gamma_{i}:=\left\{\left(\gamma_{i}(t), t\right): t>0\right\}(i=1, \ldots, N)$ be smooth curves in $\mathbb{R} \times \mathbb{R}_{+}$. Then $u \in C^{1}\left(\mathbb{R} \times \mathbb{R}_{+} \backslash \bigcup_{i=1}^{N} \Gamma_{i}\right)$ is an entropy solution of (1.1) if and only if

(i) $u$ is a classical solution of (1.1) in $\mathbb{R} \times \mathbb{R}_{+} \backslash \bigcup_{i=1}^{N} \Gamma_{i}$;

(ii) for any $t>0, i=1, \ldots, N$, the Rankine-Hugoniot condition holds:

$$
\frac{d \gamma_{i}}{d t}(t)=\frac{f\left(u_{i}^{+}(t)\right)-f\left(u_{i}^{-}(t)\right)}{u_{i}^{+}(t)-u_{i}^{-}(t)}
$$

where $u_{i}^{ \pm}(t):=\lim _{\varepsilon \rightarrow 0^{+}} u\left(t, \gamma_{i}(t) \pm \varepsilon\right)$

(iii) for any $t>0, i=1, \ldots, N$, the Oleinik condition holds:

$$
\left(s-u_{i}^{-}(t)\right)\left(f(s)-\frac{d \gamma_{i}}{d t}(t)\left(s-u_{i}^{-}(t)\right)-f\left(u_{i}^{-}(t)\right)\right) \geq 0 \quad \forall s \in \mathcal{I}\left(u_{i}^{-}(t), u_{i}^{+}(t)\right),
$$

where $\mathcal{I}(a, b):=(\min \{a, b\}, \max \{a, b\})(a, b \in \mathbb{R})$.

This criterion suggests we should look for travelling waves with a finite number of possible jump points.

Definition 2.1. An entropy travelling wave for Eq. (1.1) is an entropy solution $u=$ $u(x, t)$ of the form

$$
u(x, t)=\phi(x-c t)
$$


where $\phi$ is a monotone function belonging to $C^{1}\left(\mathbb{R} \backslash\left\{\xi_{1}, \ldots, \xi_{N}\right\}\right)$ for some $\xi_{1}, \ldots, \xi_{N}$. The function $\phi$ is called the profile of the wave, and $c$ is called the speed of the wave.

It follows at once from the previous criterion that a function $u$ of the form (2.3) is an entropy solution if and only if $\phi$ satisfies the differential equation

$$
\left(f^{\prime}(\phi)-c\right) \phi^{\prime}=g(\phi)
$$

where it is regular and the Rankine-Hugoniot and Oleinik jump conditions hold at any point $\xi_{i}(i=1, \ldots, N)$. Moreover, we look for heteroclinic travelling waves, i.e., waves satisfying the additional property

$$
\lim _{\xi \rightarrow \pm \infty} \phi(\xi)=\phi_{ \pm} \in\{0,1\} \quad \text { with } \phi_{-} \neq \phi_{+} .
$$

This requirement is natural when considering the problem of interaction between two constant states of the equation. The values $\phi_{ \pm}$are referred to as the asymptotic states of the travelling wave $\phi$.

Now we can state the result on existence of heteroclinic waves for (1.1).

Theorem 2.2. Let assumptions $(\mathrm{F})$ and $(\mathrm{G})$ be satisfied and let $c_{*}$ be defined by

$$
c_{*}:=\inf _{s \in(0,1]} \frac{f(s)}{s} .
$$

Then there exist heteroclinic travelling waves with $\phi_{-}=0$ and $\phi_{+}=1$ if and only if $c \leq c_{*}$.

Moreover, fixing the speed $c$, the waves are unique (up to translations).

Note that the extremal speed $c_{*}$ depends on the function $g$, solely through the zeroes 0 and 1 and the sign of $g$ in between. Moreover, since $f^{\prime}(0)=0, c_{*} \leq 0$.

A similar result can be proved for heteroclinic waves with asymptotic states $\phi_{-}=1$ and $\phi_{+}=0$. In this case we have existence if and only if the speed $c$ is greater than or equal to $c^{*}$, where

$$
c^{*}:=\sup _{s \in(0,1]} \frac{f(s)}{s} \geq 0 .
$$

The proof of Theorem 2.2 is constructive. Thus, as a byproduct, we obtain a result concerning the structure and the regularity of the waves. As stated below, there is a critical speed separating continuous profiles from discontinuous ones.

Theorem 2.3. Let $(\mathrm{F})$ and $(\mathrm{G})$ hold.

Let $c_{0}:=\min \left\{f_{ \pm}^{\prime}(s): s \in[0,1]\right\} \leq c_{*}$ and let $\phi_{c}$ be a travelling profile with $\phi_{-}=0$, $\phi_{+}=1$, and speed $c$.

(i) If $c<c_{0}$, then $\phi_{c} \in C^{1}(\mathbb{R})$.

(ii) If $c_{0} \leq c \leq c_{*}$, then $\phi_{c} \in C(\mathbb{R} \backslash D) \cap C^{1}(\mathbb{R} \backslash C \cup D)$, where $D$ and $C$ are finite disjoint sets.

Moreover, $\operatorname{supp} \phi_{c}$ is bounded from below if and only if $c=c_{*}$ and

$$
\left\{\begin{array}{l}
\text { either } f(\sigma)=c_{*} \sigma \text { for some } \sigma>0, \\
\text { or } c_{*}=0 \text { and } f^{\prime} / g \in L^{1}(0, \varepsilon) \text { for some } \varepsilon>0 .
\end{array}\right.
$$


Now we examine the qualitative behavior of entropy solutions of (2.1). In particular, we analyze interaction between the two constant solutions of (1.1). To this end, we consider the Riemann initial datum, given by

$$
u_{0}^{R}(x):= \begin{cases}0 & x<0 \\ 1 & x \geq 0\end{cases}
$$

We make the following additional assumption:

$$
\left\{\begin{array}{l}
\text { either } f(\sigma)=c_{*} \sigma \text { for some } \sigma>0, \\
\text { or } c_{*}=0 \text { and } f^{\prime} / g \in L^{1}(0, \varepsilon) \text { for some } \varepsilon>0 .
\end{array}\right.
$$

Note that if $f$ is twice differentiable at $0, g^{\prime}(0)$ exists and is nonzero, then assumption $(\mathrm{H})$ is satisfied.

As stated in Theorem 2.3, $(\mathrm{H})$ holds if and only if the wave with speed $c_{*}$ has support bounded from below.

In the convex case, since $f(s) / s$ is increasing, $c_{*}=f^{\prime}(0)=0$; in the concave case, $f(s) / s$ is decreasing and $c_{*}=f(1)$. These are exactly the speeds of the limiting profile of the solutions to the Riemann problem in the corresponding cases. The following result shows that the same behavior holds also in the nonconvex case.

Theorem 2.4. Assume $(\mathrm{F}),(\mathrm{G})$, and $(\mathrm{H})$. Let $u^{R}=u^{R}(x, t)$ be the entropy solution of problem (2.1) with $u_{0}$ given by (2.6) and let $\phi_{*}=\phi_{*}(\xi)$ be the travelling profile for (1.1) with asymptotic states $\phi_{-}=0, \phi_{+}=1$, speed $c_{*}$, and $\operatorname{supp} \phi_{*}=[0,+\infty)$.

Then, for any $\varepsilon>0$, there exists a set $N_{\varepsilon}$ such that

(i) meas $N_{\varepsilon}<\varepsilon$,

(ii)

$$
\lim _{t \rightarrow+\infty}\left\|u^{R}\left(\cdot+c_{*} t, t\right)-\phi_{*}(\cdot)\right\|_{L^{\infty}\left(\mathbb{R} \backslash N_{\varepsilon}\right)}=0 .
$$

If, in addition, $1-\phi_{*} \in L^{p}(a,+\infty)$ for some $a \in \mathbb{R}$, then

$$
\lim _{t \rightarrow+\infty}\left\|u^{R}(\cdot, t)-\phi_{*}\left(\cdot-c_{*} t\right)\right\|_{L^{\prime \prime}(\mathbb{R})}=0 .
$$

Finally, if $f^{\prime}(s)>0$ for any $s \in(0,1]$, the convergence in $(2.7)$ is uniform over $\mathbb{R}$.

Hypothesis $(\mathrm{H})$ is necessary in order to get (2.7). In fact, we can also prove that if (H) does not hold, then the solution of problem (2.1), (2.6) converges to zero uniformly in subsets of $\mathbb{R}$ bounded from above. The precise statement is the following.

Theorem 2.5. Assume (F) and $(\mathrm{G})$. Let $u=u(x, t)$ be the entropy solution of problem (2.1), with $u_{0} \leq u_{0}^{R}$. Moreover, suppose $f(s)>0$ for any $s>0$ and $f^{\prime} / g \notin L^{1}(0, \varepsilon)$ for any $\varepsilon>0$.

Then

$$
\lim _{t \rightarrow+\infty}\|u(\cdot, t)\|_{L^{\infty}(-\infty, M)}=0, \quad \forall M \in \mathbb{R} .
$$

Note that the assumptions of Theorem 2.5 imply that $c_{*}=f^{\prime}(0)=0$. Thus the solution cannot converge to an entropy travelling wave in the sense of Theorem 2.4.

This phenomenon can be read in the following way. Suppose $f(s) \sim s^{\alpha+1}$ and $g(s) \sim$ $s^{\beta}$ for $s \in(0, \varepsilon)$ for some $\alpha, \beta, \varepsilon>0$. Then the assumptions of Theorem 2.5 are satisfied 
if and only if $\alpha<\beta$. Hence if 0 , as a zero of $g$, is "strong" (i.e., its multiplicity $\beta$ is large) and the speed of propagation of small values of $u$, given by $f^{\prime}$, is "large" (i.e., the multiplicity $\alpha$ of 0 as zero of $f^{\prime}$ is small), then we cannot expect convergence to a travelling wave, but to zero, since the convective term rapidly moves small values of $u$ to the right.

We conclude this section with some remarks on the multi-dimensional case. As noted in [27], the analysis of multi-dimensional scalar conservation laws immediately leads one to consider the nonconvex case for one-dimensional equations. Indeed, let us consider the scalar equation

$$
\frac{\partial u}{\partial t}(\mathbf{x}, t)+\nabla_{\mathbf{x}} \cdot F(u(\mathbf{x}, t))=g(u(\mathbf{x}, t)), \quad \mathbf{x} \in \mathbb{R}^{N}, t>0,
$$

where $N \in \mathbb{N}$ and $F: \mathbb{R} \rightarrow \mathbb{R}^{N}$ is given by the vector $\left(F_{1}, \ldots, F_{N}\right)$.

Given a unit vector $\mathbf{k}=\left(k_{1}, \ldots, k_{N}\right) \in S^{N-1} \subset \mathbb{R}^{N}$, consider the Riemann initial data for $(2.9)$

$$
u(\mathbf{x}, 0)=u_{0}(\mathbf{x}):= \begin{cases}0 & \mathbf{k} \cdot \mathbf{x}<0 \\ 1 & \mathbf{k} \cdot \mathbf{x} \geq 0\end{cases}
$$

where the dot represents the usual scalar product in $\mathbb{R}^{N}$.

In order to study the asymptotic behavior, it is natural to look for planar travelling waves of $(2.9)$, i.e., solutions of the form

$$
u(\mathbf{x}, t)=\Phi(\mathbf{k} \cdot \mathbf{x}-c t) \quad(c \in \mathbb{R}),
$$

with asymptotic states given by

$$
\lim _{\xi \rightarrow-\infty} \Phi(\xi)=0, \quad \lim _{\xi \rightarrow+\infty} \Phi(\xi)=1 .
$$

Substituting (2.11) in (2.9), we get the ordinary differential equation for the profile $\Phi$ :

$$
\left(\sum_{i=1}^{N} k_{i} F_{i}^{\prime}(\Phi)-c\right) \Phi^{\prime}=g(\Phi) .
$$

Thus the existence problem for travelling profiles for (2.9) is the same for the onedimensional equation

$$
\frac{\partial u}{\partial t}+\frac{\partial f(u)}{\partial x}=g(u)
$$

with flux

$$
f(s):=\sum_{i=1}^{N} k_{i} F_{i}(s) \equiv F(s) \cdot \mathbf{k} .
$$

Hence no convexity assumptions on the functions $F_{i}$ may lead to convexity properties of the function $f$ given by $(2.12)$.

Our results on existence of entropy travelling waves can be easily applied to obtain existence of planar travelling waves for the multi-dimensional case in any direction $\mathbf{k}$ such that the function $f$, defined in (2.12), satisfies (F). 
The critical speed obviously depends on $\mathbf{k}$, and precisely is given by

$$
c_{\mathbf{k}}:=\inf _{s \in(0.1]} \frac{F(s)-F(0)}{s} \cdot \mathbf{k} .
$$

Now we turn our attention to the large-time behavior of the solution of the Riemann problem (2.9), (2.10). The symmetry of the initial data suggests constructing the solution as follows. Let $u^{R}$ be the solution of the one-dimensional Cauchy problem:

$$
\left\{\begin{array}{l}
\frac{\partial v}{\partial t}+\frac{\partial f(v)}{\partial x}=g(v), \quad(x, t) \in \mathbb{R} \times \mathbb{R}_{+}, \\
v(x, 0)= \begin{cases}0 & x<0, \\
1 & x>0,\end{cases}
\end{array}\right.
$$

with $f$ as in (2.12). It is easy to see that the unique entropy solution of $(2.9),(2.10)$, say $u_{\mathbf{k}}=u_{\mathbf{k}}(\mathbf{x}, t)$, can be written as

$$
u_{\mathbf{k}}(\mathbf{x}, t)=u^{R}(\mathbf{k} \cdot \mathbf{x}, t)
$$

Applying the results on convergence to travelling profiles of $u^{R}$, we get the convergence of the solution $u_{\mathbf{k}}$ to the travelling planar wave having profile $\Phi$ with speed $c_{\mathbf{k}}$ and support $[0,+\infty)$.

3. Entropy travelling waves. In this section we establish some results on existence and local structure of entropy travelling waves for (1.1). We consider possibly discontinuous solutions of ordinary differential equations satisfying Rankine-Hugoniot and Oleinik conditions at the jump points. Precisely, the profile $\phi$ of a travelling wave is such that for an appropriate choice of $\xi_{1}, \ldots, \xi_{N} \in \mathbb{R}$,

(a) if $\xi \notin\left\{\xi_{1}, \ldots, \xi_{N}\right\}$, then

$$
\left(f^{\prime}(\phi(\xi))-c\right) \phi^{\prime}(\xi)=g(\phi(\xi))
$$

(b) if $\xi \in\left\{\xi_{1}, \ldots, \xi_{N}\right\}$, then

$$
\begin{gathered}
c\left(\phi^{r}-\phi^{l}\right)=f\left(\phi^{r}\right)-f\left(\phi^{l}\right), \\
\left(s-\phi^{l}\right)\left[f(s)-c\left(s-\phi^{l}\right)-f\left(\phi^{l}\right)\right] \geq 0, \quad \forall s \in \mathcal{I}\left(\phi^{l}, \phi^{r}\right),
\end{gathered}
$$

where $\phi^{l}:=\lim _{\varepsilon \rightarrow 0^{+}} \phi(\xi-\varepsilon)$ and $\phi^{r}:=\lim _{\varepsilon \rightarrow 0^{+}} \phi(\xi+\varepsilon)$.

These properties suggest the following definition.

Definition 3.1. A function $\psi: I \equiv I_{\delta}\left(\xi_{0}\right):=\left\{\xi \in \mathbb{R}:\left|\xi-\xi_{0}\right|<\delta\right\} \rightarrow \mathbb{R}$ piecewise $C^{1}$ is an entropy solution of the ordinary differential equation

$$
\left(f^{\prime}(\psi)-c\right) \psi^{\prime}=g(\psi)
$$

if

(i) for any $\xi \in I$, such that there exists $J=I_{\eta}(\xi) \subset I$ with $\psi \in C^{1}(J)$, Eq. (3.1) is satisfied;

(ii) for any $\xi \in I$, such that $\psi \notin C^{1}\left(I_{\eta}(\xi)\right)$ for any $\eta>0$,

$$
c=\sigma\left(\psi^{r}, \psi^{l}\right)
$$


where

$$
\sigma\left(\psi^{r}, \psi^{l}\right):= \begin{cases}\frac{f\left(\psi^{r}\right)-f\left(\psi^{l}\right)}{\psi^{r}-\psi^{l}} & \text { if } \psi^{r} \neq \psi^{l} \\ f^{\prime}\left(\psi^{r}\right) & \text { if } \psi^{r}=\psi^{l}\end{cases}
$$

and

$$
\left(\psi^{r}-\psi^{l}\right)\left\{f(s)-\left[c\left(s-\psi^{r}\right)+f\left(\psi^{r}\right)\right]\right\} \geq 0 \quad \forall s \in \mathcal{I}\left(\psi^{l}, \psi^{r}\right),
$$

where $\mathcal{I}(a, b):=(\min \{a, b\}, \max \{a, b\})$.

An entropy solution of (3.1) defines locally an entropy solution of (1.1) by

$$
u(x, t):=\psi(x-c t) \text { for } x-c t \in I_{\delta}\left(\xi_{0}\right) .
$$

Conversely, if $u$ is a piecewise smooth entropy solution of (1.1) of the form (3.2), then $\psi$ is an entropy solution of (3.1).

In the following lemma, we assume $g>0$. This hypothesis guarantees uniqueness of the entropy solution of (3.1). We will apply these properties to obtain uniqueness of profiles of travelling waves.

It follows at once from the classical theory for ordinary differential equations that there exists locally a unique classical solution of $(3.1)$ such that $\psi\left(\xi_{0}\right)=\psi_{0}$ if $f^{\prime}\left(\psi_{0}\right) \neq c$ $\left(\xi_{0} \in \mathbb{R}\right)$. A detailed analysis of the integral curves of $(3.1)$ shows that, if $f^{\prime}\left(\psi_{0}\right)=c$, there is no local solution to the same problem. Thus the possibility of having a global solution is due to the fact that it may be discontinuous.

LEMma 3.2. Let $\psi$ be an entropy solution of $(3.1)$ in $I:=\left(\xi_{1}, \xi_{2}\right)$ such that $\psi \in$ $C^{1}\left(I \backslash\left\{\xi_{0}\right\}\right)$ for some $\xi_{0} \in I$.

Then, if $f_{-}^{\prime}(s)>c$, for $s$ in a left neighborhood of $\psi\left(\xi_{2}-\right)$, the solution $\psi$ is uniquely determined by $\psi\left(\xi_{2}-\right)$ and it is monotone.

Proof. If $\psi \in C^{1}(I)$, the statement is a consequence of the classical theory for ordinary differential equations.

Let $\psi \notin C^{1}(I)$. Set $\psi^{ \pm}:=\psi\left(\xi_{0} \pm 0\right)$. Then $\psi^{+}$is uniquely determined by $\psi\left(\xi_{2}-\right)$. Since $f_{-}^{\prime}(s)>c$ at the left of $\psi\left(\xi_{2}-\right), \psi$ is increasing in $\left(\xi_{0}, \xi_{2}\right)$. Then $f^{\prime}(s)>c$ for any $s \in\left(\psi^{+}, \psi\left(\xi_{2}-\right)\right)$. The value $\psi^{-}$belongs to $\mathcal{S}$, where

$\mathcal{S}:=\left\{\sigma \in\left[0, \psi^{+}\right]: f(\sigma)=f\left(\psi^{+}\right)+c\left(\sigma-\psi^{+}\right), f(s) \geq f\left(\psi^{+}\right)+c\left(s-\psi^{+}\right) \forall s \in\left(\sigma, \psi^{+}\right)\right\}$.

Note that $\mathcal{S} \neq \emptyset$ since $\psi^{+} \in \mathcal{S}$.

Let $\sigma_{0}:=\min \mathcal{S}$. Then $\psi^{-}=\sigma_{0}$. Indeed assume, by contradiction, $\psi^{-} \in \mathcal{S} \backslash\left\{\sigma_{0}\right\}$. By definition of $\mathcal{S}$, in a neighborhood of $\psi^{-}$, either $\left(f^{\prime}(s)-c\right)\left(s-\psi^{-}\right) \geq 0$ for $s \geq \psi^{-}$, or $f(s)=c s+$ constant. In both cases such an $f$ does not define $\psi$ in any left neighborhood of $\xi_{0}$.

Next we turn our attention to the construction of travelling waves.

Lemma 3.3. Given a compact set $K \subset \mathbb{R}$, a function $f \in \operatorname{Lip}(K)$ with Lipschitz constant $L$ and a constant $c \in \mathbb{R}$, set

$$
f_{c}(x):=\min _{y \geq x, y \in K}(f(y)-c y)+c s, \quad \forall x \in K .
$$

Then the function $f_{c}$ satisfies the following properties: 
(i) for any $x_{1}, x_{2} \in K$ such that $x_{1}<x_{2}$, it follows that

$$
c\left(x_{2}-x_{1}\right) \leq f_{c}\left(x_{2}\right)-f_{c}\left(x_{1}\right) \leq \max \{L, c\}\left(x_{2}-x_{1}\right) ;
$$

(ii) for any $x \in K, f_{c}(x) \leq f(x)$.

Note that property (i) of Lemma 3.3 implies that the function $f_{c}(x)-c x$ is monotone increasing.

Proof. (i) Let $x_{1}, x_{2} \in K$ be such that $x_{1}<x_{2}$. By definition of $f_{c}$, we get

$$
f_{c}\left(x_{2}\right)-f_{c}\left(x_{1}\right)=\min _{y \geq x_{2}}(f(y)-c y)-\min _{y \geq x_{1}}(f(y)-c y)+c\left(x_{2}-x_{1}\right) \geq c\left(x_{2}-x_{1}\right) .
$$

If $\min _{y \geq x_{2}}(f(y)-c y)=\min _{y \geq x_{1}}(f(y)-c y)$, the conclusion follows. If this is not the case, then

$$
\begin{aligned}
f_{c}\left(x_{2}\right) & -f_{c}\left(x_{1}\right) \leq f\left(x_{2}\right)-\min _{x_{1} \leq y \leq x_{2}}(f(y)-c y)-c x_{1} \\
& =f\left(x_{2}\right)-f(z)+c\left(z-x_{1}\right) \leq L\left(x_{2}-z\right)+c\left(z-x_{1}\right)
\end{aligned}
$$

for some $z \in\left[x_{1}, x_{2}\right] \cap K$. Taking the maximum with respect to $z$,

$$
f_{c}\left(x_{2}\right)-f_{c}\left(x_{1}\right) \leq \max \{L, c\}\left(x_{2}-x_{1}\right) .
$$

(ii) By definition of $f_{c}$,

$$
f_{c}(x)-c x=\min _{y \geq x}(f(y)-c y) \leq f(x)-c x .
$$

This proof is complete.

The main idea in the construction of the travelling wave is to replace the flux function $f$ with the function $f_{c}$. The monotonicity of $f_{c}(s)-c s$ permits us to construct the inverse of the profile of the wave. This function turns out to be regular and monotone; thus, after an inversion, it gives the desired travelling wave.

In order to prove the result on the regularity of the wave and of the structure of the set of jumps, we need some more information on the function $f_{c}$.

Lemma 3.4. Let $f$ and $f_{c}$ be as in Lemma 3.3. Let $x_{0} \in K$ be such that $f_{c}\left(x_{0}\right)<f\left(x_{0}\right)$ and let $I$ be the connected component of $\left\{x \in K: f_{c}(x)<f(x)\right\}$ containing $x_{0}$.

Then

$$
f_{c}(x)-c x=f_{c}\left(x_{0}\right)-c x_{0}, \quad \forall x \in I .
$$

Proof. Let $J:=\left\{x \in I: f_{c}(x)-c x=f_{c}\left(x_{0}\right)-c x_{0}\right\}$. Then $J$ is a closed set in $I$. We claim that $J$ is also an open set in $I$.

Assume, by contradiction, that this is not the case. Then there exist $x^{*} \in J$ and $x_{n} \notin J(n \in \mathbb{N})$ such that $x_{n} \rightarrow x^{*}$ as $n \rightarrow \infty$. Then

$$
f_{c}\left(x^{*}\right)-c\left(x^{*}\right)=f_{c}\left(x_{0}\right)-c x_{0}, \quad f_{c}\left(x_{n}\right)-c\left(x_{n}\right) \neq f_{c}\left(x_{0}\right)-c x_{0} \quad(\forall n) .
$$

Assume $x_{n}<x^{*}$ for any $n$ (the general case is similar). Since $f_{c}(x)-c x$ is an increasing function,

$$
\min _{y \geq x_{n}}(f(y)-c y)=f_{c}\left(x_{n}\right)-c x_{n}<f_{c}\left(x^{*}\right)-c x^{*}=\min _{y \geq x^{*}}(f(y)-c y) .
$$


Then, for any $n \in \mathbb{N}$, there exists $z_{n} \in\left[x_{n}, x^{*}\right)$ such that $f\left(z_{n}\right)-c z_{n}=\min _{y \geq x_{n}}(f(y)-$ $c y$ ), implying $f\left(z_{n}\right)=f_{c}\left(z_{n}\right)$. Passing to the limit as $n \rightarrow \infty$, it follows that $f\left(x^{*}\right)=$ $f_{c}\left(x^{*}\right)$, contradicting the assumption $x^{*} \in I$.

Since $J$ is both closed and open in $I$, the conclusion follows.

LEMma 3.5. Let $f$ and $f_{c}$ be as in Lemma 3.3 with $K=[0,1]$. Suppose that $f$ satisfies (F). Finally, let $I=\left(s_{1}, s_{2}\right)$ be such that

$$
f_{c}(s)=f_{c}\left(s_{1}\right)+c\left(s-s_{1}\right)
$$

for any $s \in I$ and such that (3.3) is false for any open set $J$ containing $I$.

Then

(i) $f_{c}\left(s_{2}\right)=f\left(s_{2}\right)$ and, if $s_{1} \neq 0, f_{c}\left(s_{1}\right)=f\left(s_{1}\right)$;

(ii) $f_{-}^{\prime}\left(s_{2}\right) \leq c \leq f_{+}^{\prime}\left(s_{2}\right)$;

(iii) $a_{i} \in\left[s_{1}, s_{2}\right]$ for some $i \in\{0, \ldots, n-1\}$.

Proof. (i) It is a consequence of Lemma 3.4, and of $f_{c}(1)=f(1)$.

(ii) It follows that

$$
f\left(s_{2}\right)-c s_{2}=\min _{s \geq s_{1}}(f(s)-c s) .
$$

Indeed, $f_{c}\left(s_{2}\right)=f\left(s_{2}\right)$ implies $f\left(s_{2}\right)-c s_{2}=\min _{s \geq s_{2}}(f(s)-c s)$. Moreover, for any $\sigma \in\left[s_{1}, s_{2}\right)$,

$$
\begin{aligned}
f(\sigma)-c \sigma \geq f_{c}(\sigma)-c \sigma & =f_{c}\left(s_{1}\right)+c\left(\sigma-s_{1}\right)-c \sigma \\
& =f_{c}\left(s_{1}\right)+c\left(s_{2}-s_{1}\right)-c s_{2} \\
& =f_{c}\left(s_{2}\right)-c s_{2}=f\left(s_{2}\right)-c s_{2} .
\end{aligned}
$$

Hence

$$
f\left(s_{2}\right)-c s_{2}=\min _{s \in\left[s_{1}, s_{2}\right]}(f(s)-c s)
$$

Since $s_{2}$ is a minimum point for the function $f(s)-c s$, the conclusion follows.

(iii) Suppose by contradiction that $a_{i} \notin\left[s_{1}, s_{2}\right]$ for any $i$. Then $f \in C^{1}\left(\left[s_{1}, s_{2}\right]\right)$. If $f_{c} \equiv f$ over all $I$, the result is obvious. Now suppose that there is $s_{3} \in\left(s_{1}, s_{2}\right)$ such that $f_{c}\left(s_{3}\right)<f\left(s_{3}\right)$. Then there are $s_{4}$ and $s_{5}$ such that $s_{1}<s_{4}<s_{3}<s_{5}<s_{2}$ and

$$
\begin{aligned}
f^{\prime}\left(s_{4}\right) & =\frac{f\left(s_{3}\right)-f\left(s_{1}\right)}{s_{3}-s_{1}}>\frac{f_{c}\left(s_{3}\right)-f_{c}\left(s_{1}\right)}{s_{3}-s_{1}}=c, \\
f^{\prime}\left(s_{5}\right) & =\frac{f\left(s_{2}\right)-f\left(s_{3}\right)}{s_{2}-s_{3}}<\frac{f_{c}\left(s_{2}\right)-f_{c}\left(s_{3}\right)}{s_{2}-s_{3}}=c .
\end{aligned}
$$

Therefore, there exist $s_{4}<s_{5}<s_{2}$ such that

$$
f^{\prime}\left(s_{4}\right)>f^{\prime}\left(s_{5}\right) \text { and } f^{\prime}\left(s_{5}\right)<f^{\prime}\left(s_{2}\right) .
$$

Since $f^{\prime}$ is nonmonotonic in $I, f$ has an inflection point at some $s_{0} \in I$. Thus $s_{0}=a_{i}$ for some $i$.

Proof of Theorem 2.2. Let $c \leq c_{*}$.

By definition of $f_{c}$, it follows that $f_{c}^{\prime}(s) \geq c$ a.e. 
Therefore, given $s_{0} \in(0,1)$, the following function is well defined:

$$
\Xi(s):=\int_{s_{0}}^{s} \frac{f_{c}^{\prime}(\sigma)-c}{g(\sigma)} d \sigma, \quad \forall s \in(0,1),
$$

and the derivative of $\Xi$ is

$$
\frac{d \Xi}{d s}=\frac{f^{\prime}(s)-c}{g(s)} \geq 0 \quad \text { a.e. }
$$

Set $\xi_{0}:=\Xi(0)$ and $\xi_{1}:=\Xi(1)$. Then

$$
-\infty \leq \xi_{0} \leq 0 \leq \xi_{1} \leq+\infty
$$

and

$$
\left\{\begin{array}{l}
\xi_{0}>-\infty \text { if and only if } \frac{f_{c}^{\prime}-c}{g} \in L^{1}(0, \varepsilon) \text { for some } \varepsilon>0, \\
\xi_{1}<+\infty \text { if and only if } \frac{f_{c}^{\prime}-c}{g} \in L^{1}(1-\varepsilon, 1) \text { for some } \varepsilon>0 .
\end{array}\right.
$$

From hypothesis $(\mathrm{F})$ and Lemma 3.5(iii), there exist at most finitely many intervals, say $\left[s_{1}, t_{1}\right], \ldots,\left[s_{N}, t_{N}\right]$, such that

$$
\frac{d \Xi}{d s}(s) \equiv \frac{f_{c}^{\prime}(s)-c}{g(s)}=0 \quad \forall s \in \bigcup_{i=1}^{N}\left[s_{i}, t_{i}\right] .
$$

Next, we define the following multi-valued function:

$$
\hat{\phi}(\xi):= \begin{cases}0, & \xi \leq \xi_{0}, \\ \Xi^{-1}(\xi), & \xi_{0}<\xi<\xi_{1}, \\ 1, & \xi \geq \xi_{1} .\end{cases}
$$

If the function $\hat{\phi}$ is multi-valued at $\bar{\xi} \in\left(\xi_{0}, \xi_{1}\right)$, then

$$
\Xi^{-1}(\xi) \in \bigcup_{i=1}^{N}\left[s_{i}, t_{i}\right]
$$

Set

$$
\xi_{i}:=\Xi^{-1}\left(s_{i}\right), \quad i=1, \ldots, N .
$$

Then we define the profile $\phi$ of the wave:

$$
\phi(\xi):= \begin{cases}\hat{\phi}(\xi), & \xi \in \mathbb{R} \backslash\left\{\xi_{1}, \ldots, \xi_{N}\right\} \\ s_{i}, & \xi=\xi_{i}, i=1, \ldots, N .\end{cases}
$$

If $s_{i} \neq t_{i}$, then $\phi$ is discontinuous at $\xi_{i}$ and

$$
\phi\left(\xi_{i}-\right)=s_{i}, \quad \phi\left(\xi_{i}+\right)=t_{i} .
$$

If $s_{i}=t_{i}$, then $\phi$ is continuous at $\xi_{i}$, but not differentiable.

If $s \notin \bigcup\left(s_{i}, t_{i}\right)$, then $f_{c}(s)=f(s)$. Thus,

$$
\frac{d \Xi}{d s}=\frac{f^{\prime}(\phi)-c}{g(\phi)}
$$

and (3.1) is satisfied.

If $s \in\left(s_{i}, t_{i}\right)$ for some $i$ and $s_{i} \neq t_{i}$, the wave $\phi$ is discontinuous. We have to check that conditions (RH) and $(\mathrm{O})$ hold. 
Let $s_{i} \neq 0$. Then $(\mathrm{RH})$ is a consequence of Lemma 3.5(i); indeed,

$$
\frac{f\left(s_{i}\right)-f\left(t_{i}\right)}{s_{i}-t_{i}}=\frac{f_{c}\left(s_{i}\right)-f_{c}\left(t_{i}\right)}{s_{i}-t_{i}}=c .
$$

Moreover, $f(s)-f_{c}(s) \geq 0$. Since, for any $s \in\left(s_{i}, t_{i}\right), f_{c}(s)=f\left(t_{i}\right)+c\left(s-t_{i}\right)$, we get

$$
f(s)-f\left(t_{i}\right)-c\left(s-t_{i}\right) \geq 0 \quad \forall s \in\left(s_{i}, t_{i}\right),
$$

that is, exactly $(\mathrm{O})$.

Now suppose that $s_{i}=0$. Then $c=c_{*}$. In fact, let $c<c_{*}$. Then

$$
f(s)-c s>0 \quad \forall s \in[0,1] .
$$

But, at $t_{i}$, we have $f\left(t_{i}\right)=c t_{i}$, that contradicts (3.5).

Thus, it remains to show that if $c=c_{*}$ and $s_{i}=0$, the jump interval $\left(s_{i}, t_{i}\right)$ is admissible. Condition (O) can be proved as in the case $s_{i}>0$. For the Rankine-Hugoniot condition we have to check that

$$
c_{*} \equiv \frac{f_{c}\left(t_{i}\right)-f_{c}(0)}{t_{i}-0}=\frac{f\left(t_{i}\right)}{t_{i}} .
$$

By Lemma 3.5(i), $f_{c}\left(t_{i}\right)=f\left(t_{i}\right)$; thus, we only have to show that $f_{c}(0)=0$. Since $f_{c}(0) \leq f(0)=0$, it is enough to prove $f_{c}(0) \geq 0$. From $c \leq c_{*}$, we deduce $c s \leq f(s)$ for any $s \in[0,1]$. Hence $f_{c}(0)=\min _{s \in[0,1]}(f(s)-c s) \geq 0$. Then $f_{c}(0)=0$ and the conclusion follows.

Now we prove that for $c>c_{*}$, there is no travelling wave with asymptotic states 0 and 1. On the contrary, let $\phi$ be such an entropy travelling wave with speed $c$. By definition of $c_{*}$, there exists $s \in[0,1]$ such that

$$
f(s)<c s .
$$

Let

$$
s_{0}:=\inf \left\{s \in[0,1]: f(s)-c s=f_{c}(0)\right\} .
$$

If $s_{0}=0$, then $f(t)-c t \geq 0$ for any $t>0$, contradicting (3.6). Hence $s_{0}>0$. There exists $\delta>0$ such that

$$
\left(f^{\prime}(s)-c\right)\left(s-s_{0}\right) \geq 0 \quad \forall s \in I_{\delta}\left(s_{0}\right) .
$$

There is no local classical solution of (3.1) satisfying $\psi\left(\xi_{0}\right)=s_{0}$. Thus there is $\xi_{0} \in \mathbb{R}$ and $s_{1} \leq s_{0} \leq s_{2}$ such that the wave $\phi$ is discontinuous in $\xi_{0}$ and it has left and right limits at $\xi_{0}$ the values $s_{1}$ and $s_{2}$ respectively.

Since $f(s)-c s$ has minimum at $s_{0}$, condition $(\mathrm{O})$ implies

$$
f\left(s_{1}\right)-c s_{1}=f\left(s_{2}\right)-c s_{2}=f\left(s_{0}\right)-c s_{0}=\min _{s \in[0,1]}(f(s)-c s) .
$$

From the definition of $s_{0}$, it follows that $s_{1}=s_{0}$. Then we get a contradiction from the fact that the integral curves of (3.1) are decreasing at the left of $\xi_{0}$, and thus $\phi(\xi) \geq s_{0}>0$ for any $\xi \in \mathbb{R}$.

To prove uniqueness, first of all, let us suppose $f^{\prime}(s) \leq c$ in a left neighborhood of 1. Then (3.1) does not define an increasing function converging to 1 at $+\infty$. Therefore, there exists $\xi_{0} \in \mathbb{R}$ such that $\phi\left(\xi_{0}-\right)<1=\phi\left(\xi_{0}+\right)$. Moreover, $f^{\prime}(s)<c$ in a left 
neighborhood of $\phi\left(\xi_{0}-\right)$. By Lemma 3.2, coming back from $\xi_{0}$ to $-\infty$, we deduce that the solution is uniquely determined by $\xi_{0}$ and the conclusion follows.

Let $f^{\prime}(s)>c$ in a left neighborhood of 1 . If $\phi=\phi(\xi)$ is regular for any $\xi$, uniqueness is a consequence of the results for ordinary differential equations. Otherwise there is $\xi_{0}$ such that $\phi$ is discontinuous at $\xi_{0}$. Then, applying Lemma 3.2, we obtain the uniqueness of the profile.

Proof of Theorem 2.3. Let $c<c_{0}$. Since

$$
f_{ \pm}^{\prime}(s)>c_{0} \quad \forall s \in[0,1]
$$

it follows that $f_{c} \equiv f$. By (3.7) we deduce that for the function $\xi$, given in (3.4), it follows that

$$
\frac{d \Xi}{d s}(s)>0, \quad \forall s
$$

Therefore, $\Xi$ is globally invertible and the travelling wave $\phi$ belongs to $C^{1}(\mathbb{R})$.

If $c_{0} \leq c \leq c_{*}$, then there is a finite number of points, say $\xi_{1}, \ldots, \xi_{N}$, such that

$$
\phi\left(\xi_{i}-\right)=s_{i}, \quad \phi\left(\xi_{i}+\right)=t_{i},
$$

with $s_{i}, t_{i}$ given as in the proof of Theorem 2.2 .

Set

$$
C:=\left\{\xi_{i}: s_{i}=t_{i}\right\}, \quad D:=\left\{\xi_{i}: s_{i} \neq t_{i}\right\} .
$$

Then $\phi \in C(\mathbb{R} \backslash D) \cap C^{1}(\mathbb{R} \backslash C \cup D)$.

From the definition of $\phi$, we deduce that the support of $\phi$ is bounded from below if and only if $\xi_{0} \equiv \xi(0) \geq-\infty$. This condition is equivalent to the following:

$$
\frac{f_{c}^{\prime}-c}{g} \in L^{1}(0, \varepsilon) \text { for some } \varepsilon>0 .
$$

Since $g(0)=0$, this implies that $f_{c}^{\prime}(0)=c$. Hence $s_{1}=0$ and $c=c_{*}$.

Now there are two possibilities: either $f_{c}(s)=c_{*} s$ for any $s \in[0, \sigma)$ for some $\sigma>0$ or $f_{c}(s)=f(s)$ for any $s \in[0, \sigma)$ for some $\sigma>0$. In the former case, (3.8) holds. In the latter, $c=c_{*}=f_{c}^{\prime}(0)=f^{\prime}(0)=0$, and (3.8) is equivalent to

$$
c_{*}=0, \quad f^{\prime} / g \in L^{1}(0, \varepsilon) .
$$

This completes the proof.

4. Asymptotic behavior of solutions. This section is devoted to the proof of Theorem 2.4. We always assume $(\mathrm{F}),(\mathrm{G}),(\mathrm{H})$. Let us denote with $u^{R}$ the entropy solution of the Cauchy problem $(1.1),(2.6)$ and with $f_{*}$ the function $f_{c_{*}}$, defined in Sec. 3 .

Moreover, let $\phi_{*}$ be the heteroclinic travelling wave with $\phi_{-}=0, \phi_{+}=1$, speed $c_{*}$ and supported in $[0,+\infty)$. Let $C \cup D=\left\{\xi_{1}<\cdots<\xi_{N}\right\}$ with $C$ and $D$ as in Theorem 2.3. Finally set $\xi_{N+1}=+\infty$.

Our main tools in proving large-time behavior for the Riemann problem are comparison results for Eq. (1.1). For completeness, we recall these properties of entropy solutions of balance laws (see [19]). 
Let us consider the following Cauchy problem:

$$
\begin{cases}\frac{\partial u}{\partial t}+\frac{\partial f(u)}{\partial x}=g(u) & \text { in } \mathbb{R} \times(0, T), \\ u(x, 0)=u_{0}(x) & \text { in } \mathbb{R},\end{cases}
$$

where $T>0, u_{0} \in L_{\text {loc }}^{\infty}(\mathbb{R})$.

Moreover, let us set

$$
[r]_{ \pm}:=\frac{1}{2}(|r| \pm r), \quad H_{ \pm}(r):=\frac{1}{2}(\operatorname{sgn} r \pm 1) .
$$

Definition 4.1. A measurable function $u$ is called an entropy supersolution of problem (4.1) if

(i) $u \in L_{\text {loc }}^{\infty}(\mathbb{R} \times(0, T))$;

(ii) for any nonnegative function $\eta \in C_{0}^{\infty}(\mathbb{R} \times(0, T))$ and $k \in \mathbb{R}$, it follows that

$$
\begin{array}{r}
\iint_{\mathbb{R} \times(0, T)}\left\{[u-k]-\frac{\partial \eta}{\partial t}\right. \\
+H_{-}(u-k)(f(u)-f(k)) \frac{\partial \eta}{\partial x} \\
\left.+H_{-}(u-k) g(u) \eta\right\} d x d t \geq 0 ;
\end{array}
$$

(iii) for any interval $(a, b) \subseteq \mathbb{R}$

$$
\text { ess } \lim _{t \rightarrow 0^{+}} \int_{a}^{b}\left[u(x, t)-u_{0}(x)\right]_{-} d x=0 .
$$

An entropy subsolution of problem (4.1) is defined by replacing $H_{-}(\cdot)$ be $H_{+}(\cdot)$ and $[\cdot]_{-}$ by $[\cdot]_{+}$in (ii) and (iii).

Then the following comparison result can be proved.

TheOREm 4.2 (Comparison principle). Let assumptions (F) and (G) be satisfied. If $\underline{u}$ (resp. $\bar{u}$ ) is an entropy subsolution (resp. supersolution) of problem (4.1) and if

$$
\underline{u}(x, 0) \leq \bar{u}(x, 0) \text { a.e. in } \mathbb{R},
$$

then

$$
\underline{u}(x, t) \leq \bar{u}(x, t) \quad \text { a.e. in } \mathbb{R} \times \mathbb{R}_{+} .
$$

Since we want to construct some explicit supersolution $u$ of (1.1), we proceed by considering some additional assumptions on the function $u$.

First of all, let $u=u(x, t)$ be a classical $C^{1}$ solution of (1.1) in $\mathbb{R} \times \mathbb{R}_{+} \backslash \Gamma$ where $\Gamma:=\{(\gamma(t), t): t>0\}$ and $\gamma$ is regular. Then by Definition $4.1, u$ is a supersolution if and only if

$$
\gamma^{\prime}(t)\left(\left[u^{+}-s\right]_{-}-\left[u_{-}-s\right]_{-}\right) \geq H_{-}\left(u_{+}-s\right)\left[f\left(u_{+}\right)-f(s)\right]-H_{-}\left(u_{-}-s\right)\left[f\left(u_{-}\right)-f(s)\right],
$$

for any $s \in \mathcal{I}\left(u_{+}, u_{-}\right)$and for any $t>0$, where $u_{ \pm}:=\lim _{\varepsilon \rightarrow 0^{+}} u(\gamma(t) \pm \varepsilon, t)$.

If we suppose further that $u_{-}<u_{+}$for any $t>0$, we get that $u$ is a supersolution if and only if

$$
f(s) \geq \gamma^{\prime}(t)\left(s-u_{-}\right)+f\left(u_{-}\right), \quad \forall s \in\left[u_{-}, u_{+}\right], t>0 .
$$


Let $\phi_{*}$ be the unique entropy travelling wave with asymptotic states $\phi_{-}=0, \phi_{+}=1$, speed $c_{*}$ and $\operatorname{supp} \phi_{*}=[0+\infty)$. We define, locally in time, a family of supersolutions with the above structure, where $\gamma$ is an affine function. We set

$$
V(x, t ; y, k):= \begin{cases}\phi_{*}\left(x-c_{*} t\right), & x<k t+y \\ 1, & x>k t+y\end{cases}
$$

with $x, k \in \mathbb{R}$ and $y, t>0$.

Let $K$ be defined by

$$
K(s):=\inf _{\sigma \in(s, 1]} \frac{f(\sigma)-f(s)}{\sigma-s}, \quad s \in[0,1) .
$$

Proposition 4.3. Let $y>0$ be such that $c_{*}<K\left(\phi_{*}(y)\right)$. Given $k \in\left(c_{*}, K\left(\phi_{*}(y)\right)\right)$, then the function $u(x, t):=V(x, t ; y, k)$ is an entropy supersolution of $(1.1)$ in $(0, T) \times \mathbb{R}$ with

$$
T \equiv T(y, k):=\sup \left\{t \geq 0: \forall \tau \leq t, K\left(\phi_{*}^{\tau}\right) \geq k\right\}>0,
$$

where $\phi_{*}^{t}:=\phi_{*}\left(y+\left(k-c_{*}\right) t\right)$.

Proof. For the family of functions defined by (4.3), condition (4.2) becomes

$$
f(s) \geq k\left(s-\phi_{*}^{t}\right)+f\left(\phi_{*}^{t}\right) \quad \forall s \in\left[\phi_{*}^{t}, 1\right] .
$$

Therefore, $V=V(x, t ; y, k)$ is a supersolution if and only if, for any $t \in(0, T)$, $k \leq K\left(\phi_{*}^{t}\right)$. This immediately leads to (4.5).

To complete the proof we have to show that, under the assumptions of Proposition 4.3, $T=T(y, k)>0$. We do this by proving that if $c_{*}<k<K\left(\phi_{*}(y)\right)$, then there exists $t_{0}>0$ such that $K\left(\phi_{*}^{t}\right)>k$ for any $t \in\left(0, t_{0}\right)$.

On the contrary, there is a decreasing sequence $\left\{t_{n}\right\}$ such that

$$
\lim _{n \rightarrow+\infty} t_{n}=0 \quad \text { and } K\left(\phi_{*}^{t_{n}}\right) \leq k \quad \forall n .
$$

From the definition of $K$ (see formula (4.4)), we get that there is a subsequence of $\left\{t_{n}\right\}$ (for simplicity we again denote it by $\left\{t_{n}\right\}$ ) such that either

$$
f_{+}^{\prime}\left(\phi_{*}^{t_{n}}\right)=K\left(\phi_{*}^{t_{n}}\right) \quad \forall n
$$

or

$$
\frac{f\left(\phi_{*}^{t_{n}}\right)-f\left(\sigma_{n}\right)}{\phi_{*}^{t_{n}}-\sigma_{n}}=K\left(\phi_{*}^{t_{n}}\right) \quad \forall n
$$

for some $\sigma_{n}>\phi_{*}^{t_{n}}$.

If (4.6) holds, as $n \rightarrow+\infty$, we obtain the following contradiction:

$$
K\left(\phi_{*}(y)\right) \leq f_{+}^{\prime}\left(\phi_{*}(y)\right) \leq k<K\left(\phi_{*}(y)\right)
$$

in fact, $K(s) \geq f_{+}^{\prime}(s)$ and $f_{+}^{\prime}$ is right continuous.

If (4.7) is true, then we can take a subsequence such that, for some $\sigma_{0}, \lim _{n \rightarrow+\infty} \sigma_{n}=$ $\sigma_{0}$. Then

$$
\lim _{n \rightarrow+\infty} \frac{f\left(\phi_{*}^{t_{n}}\right)-f\left(\sigma_{n}\right)}{\phi_{*}^{t_{n}}-\sigma_{n}}= \begin{cases}\frac{f\left(\phi_{*}^{t^{n}}\right)-f\left(\sigma_{0}\right)}{\phi_{*}^{t_{n}}-\sigma_{0}} & \text { if } \sigma_{0} \neq \phi_{*}(y) \\ f_{+}^{\prime}\left(\phi_{*}(y)\right) & \text { if } \sigma_{0}=\phi_{*}(y)\end{cases}
$$


Hence

$$
K\left(\phi_{*}(y)\right) \leq \lim _{n \rightarrow+\infty} \frac{f\left(\phi_{*}^{t_{n}}\right)-f\left(\sigma_{n}\right)}{\phi_{*}^{t_{n}}-\sigma_{n}} \leq k<K\left(\phi_{*}(y)\right) .
$$

The proof is complete.

Lemma 4.4. Assume the hypotheses of Proposition 4.3. Let $y \in\left(\xi_{i}, \xi_{i+1}\right)$ with $i \in$ $\{1, \ldots, n\}$. Then

$$
\lim _{k \rightarrow c_{*}^{+}} y+\left(k-c_{*}\right) T(y, k)=\xi_{i+1} .
$$

Proof. Given $\xi \in \mathbb{R}$, let $\sigma>\phi_{*}(\xi)$. Then $f_{*}\left(\phi_{*}(\xi)\right)=f\left(\phi_{*}(\xi)\right)$ and

$$
\frac{f(\sigma)-f\left(\phi_{*}(\xi)\right)}{\sigma-\phi_{*}(\xi)} \geq \frac{f_{*}(\sigma)-f_{*}\left(\phi_{*}(\xi)\right)}{\sigma-\phi_{*}(\xi)} \geq c_{*} \quad \forall \sigma>\phi_{*}(\xi) .
$$

This implies that

$$
K\left(\phi_{*}(\xi)\right) \geq c_{*} \quad \forall \xi \in \mathbb{R} .
$$

If $c_{*}<K\left(\phi_{*}(\xi)\right)$, then $f^{\prime}\left(\phi_{*}(\xi)\right)>c_{*}$. Suppose, by contradiction, that $\phi_{*}(\xi)=s_{i}$ for some $i$. Then there would exist some $t_{i}>s_{i}$ such that

$$
c_{*}=\frac{f\left(t_{i}\right)-f\left(s_{i}\right)}{t_{i}-s_{i}} \geq K(\phi(\xi))>c_{*} .
$$

If $c_{*}=K\left(\phi_{*}(\xi)\right)$, then either $f^{\prime}\left(\phi_{*}(\xi)\right)=c_{*}$ or there is $\sigma>\phi_{*}(\xi)$ such that

$$
f(\sigma)=f\left(\phi_{*}(\xi)\right)+c_{*}\left(\sigma-\phi_{*}(\xi)\right)
$$

In the first case, $\phi_{*}=s_{i}$ for some $i$. In the latter,

$$
f_{*}(\sigma)-c_{*} \sigma \leq f_{*}\left(\phi_{*}(\xi)\right)-c_{*} \phi_{*}(\xi) .
$$

By monotonicity of $f_{*}(s)-c_{*} s$ we obtain that

$$
f_{*}(s)=f_{*}\left(\phi_{*}(\xi)\right)+c_{*}\left(s-\phi_{*}(\xi)\right) \quad \forall s \in\left(\phi_{*}(\xi), \sigma\right),
$$

and $\phi_{*}(\xi)=s_{i}$ for some $i$.

Therefore,

$$
\phi_{*}(\xi) \neq s_{i} \quad \forall i \quad \text { if and only if } \quad K\left(\phi_{*}(\xi)\right)>c_{*} .
$$

Now let $\left\{k_{j}\right\}$ be a sequence converging to $c_{*}$ from the right as $j \rightarrow+\infty$ such that

$$
\lim _{j \rightarrow+\infty} y+\left(k_{j}-c_{*}\right) T\left(y, k_{j}\right)=z
$$

for some $z$. Since, for any $t<T, \xi_{i}<y \leq y+\left(k_{j}-c_{*}\right) T\left(y, k_{j}\right) \leq \xi_{i+1}$, we have

$$
\xi_{i}<y \leq z \leq \xi_{i+1} \text {. }
$$

If $K\left(\phi_{*}(z)\right)>c_{*}$, arguing as in the proof of Proposition 4.3, we can choose $k \in$ $\left(c_{*}, K\left(\phi_{*}(y)\right)\right)$ and $t_{0}>0$ such that

$$
T(y, k)+t_{0} \in\left\{t>0: \forall \tau \leq t K\left(\phi_{*}^{\tau}\right) \geq k\right\},
$$

contradiction the definition of $T(y, k)$. Thus $K\left(\phi_{*}(z)\right)=c_{*}$.

From (4.8) we get that $z=\xi_{j}$ for some $j$. Since (4.9) holds, $z=\xi_{i+1}$. 
LEMma 4.5. Let the assumptions of Proposition 4.3 be satisfied. Let $y$ be such that $\phi_{*} \in C^{1}([y,+\infty))$. Then there exists $k_{*} \geq c_{*}$ such that

$$
\lim _{k \rightarrow k_{*}^{+}} T(y, k)=+\infty
$$

Moreover, if $f^{\prime}(1) \neq c_{*}$, then $k_{*}>c_{*}$.

Proof. First of all we prove that the function $T=T(y, k)$ is decreasing with respect to $k$. Hereafter, to emphasize the dependence on $k$, we denote with $\phi_{*}^{t, k}$ the following:

$$
\phi_{*}^{k . t}:=\phi_{*}\left(y+\left(k-c_{*}\right) t\right)
$$

Moreover, set

$$
\mathcal{T}_{k}:=\left\{t \geq 0: \forall \tau \leq t K\left(\phi_{*}^{k, \tau}\right) \geq k\right\}
$$

Let $c_{*}<k_{1}<k_{2}<K\left(\phi_{*}(y)\right)$.

Let $t \in \mathcal{T}_{k_{2}}$. We will show that $t \in \mathcal{T}_{k_{2}}$. Given $\tau \leq t$, since $\phi_{*}$ is an increasing function,

$$
\phi_{*}^{0 . k_{1}}=\phi_{*}^{0 . k_{2}} \leq \phi_{*}^{\tau, k_{1}} \leq \phi_{*}^{\tau, k_{2}} .
$$

Then there exists $\theta \leq \tau$ such that $\phi_{*}^{\theta \cdot k_{2}}=\phi_{*}^{\theta \cdot k_{1}}$.

Since $t \in \mathcal{T}_{k_{2}}$ and $\theta \leq \tau$, we have $K\left(\phi_{*}^{\theta \cdot k_{2}}\right) \geq k_{2} \geq k_{1}$. Hence $K\left(\phi_{*}^{\theta \cdot k_{1}}\right) \geq k_{1}$. Then we obtain $\mathcal{T}_{k_{2}} \subseteq \mathcal{T}_{k_{1}}$ and

$$
T\left(y, k_{2}\right) \leq T\left(y, k_{1}\right) \quad \text { if } k_{2}<k_{1} .
$$

Now suppose $y$ is such that $\phi_{*} \in C^{1}([y,+\infty))$.

If there is $k>c_{*}$ such that $T(y, k)=+\infty$, the conclusion follows from (4.10) by defining $k_{*}:=\sup \left\{k>c_{*}: T(y, k)=+\infty\right\}$.

Suppose that $T(y, k)<+\infty$ for any $k>c_{*}$. We show that in this case $f^{\prime}(1)=c_{*}$. For any $k$ there exist $t_{k}<T(y, k)$ and $\tau_{k}<t_{k}$ such that $K\left(\phi_{*}^{\tau_{k} . k}\right)<k$. Therefore,

$$
k>\frac{f(s)-f\left(\phi_{*}^{\tau_{k}, k}\right)}{s-\phi_{*}^{\tau_{k} . k}} \quad \forall s>\phi_{*}^{\tau_{*}, k} .
$$

Now we pass to the limit as $k \rightarrow c_{*}^{+}$on a sequence such that $\lim \phi_{*}^{\tau_{k}, k}=\phi_{*}^{\infty}$ for some $\phi_{*}^{\infty}$. If $\phi_{*}^{\infty}<1$, then

$$
c_{*} \geq \frac{f(s)-f\left(\phi_{*}^{\infty}\right)}{s-\phi_{*}^{\infty}} \quad \forall s>\phi_{*}^{\infty} .
$$

Moreover, since $\phi_{*}$ is regular in $[y,+\infty), f(s)=f_{*}(s)$ for any $s \geq \phi_{*}^{x}$. Hence

$$
c_{*} \geq \frac{f_{*}(s)-f_{*}\left(\phi_{*}^{\infty}\right)}{s-\phi_{*}^{\infty}} \geq c_{*} .
$$

Then

$$
f_{*}(s)=f_{*}\left(\phi_{*}^{x}\right)+c_{*}\left(s-\phi_{*}^{\infty}\right) \quad \forall s \geq \phi_{*}^{x},
$$

and $\phi_{*}$ would be discontinuous. This contradicts the hypothesis on $\phi_{*}$. Thus $\phi_{*}^{\infty}=1$.

Moreover, from (4.13) with $s=1$, and passing to the limit, we get $c_{*} \geq f^{\prime}(1)$. Since $\phi_{*}$ is $C^{1}$ at $+\infty, f^{\prime}(1) \geq c_{*}$. Therefore, $f^{\prime}(1)=c_{*}$. 
To complete the proof, we have to show that, if $f^{\prime}(1)=c c_{*}$ and $T(y, k)<+\infty$ for any $k>c_{*}$, then

$$
\lim _{k \rightarrow c_{*}^{+}} T(y, k)=+\infty
$$

Suppose by contradiction that

$$
\lim _{k \rightarrow c_{*}^{+}} T(y, k)=T_{*}<+\infty .
$$

Then for any $k>c_{*}$, there is $\tau_{k} \in\left[T(y, k), 2 T_{*}\right)$ such that

$$
k>\frac{f(s)-f\left(\phi_{*}^{\tau_{k}, k}\right)}{s-\phi_{*}^{\tau_{k}, k}} \quad \forall s>\phi_{*}^{\tau_{k}, k} .
$$

Let $k \rightarrow c_{*}$. Then there are two cases: either there exists a sequence $\left\{k_{j}\right\}$ such that

$$
\lim _{j \rightarrow+\infty} \tau_{k_{j}}>0
$$

or

$$
\lim _{k \rightarrow c_{*}^{+}} \tau_{k}=0
$$

In the first case,

$$
\lim _{j \rightarrow+\infty} \phi_{*}^{\tau_{k_{j}}, k_{j}}=\phi_{*}(y)
$$

hence, by (4.12), $c_{*} \geq K\left(\phi_{*}(y)\right)$, which contradicts the choice of $y$.

In the second case, we get

$$
\lim _{k \rightarrow c_{*}^{+}} T(y, k)=0,
$$

from which we deduce $T(y, k)=0$ for any $k$. This contradicts the positivity of $T(y, k)$, stated in Proposition 4.3. Hence $T_{*}=+\infty$.

Proof of Theorem 2.4. We construct an appropriate set of supersolutions of the type (4.5).

Let $\eta$ and $k$ be such that

$$
0<\eta<\xi_{i+1}-\xi_{i} \quad \text { and } \quad c_{*}<k<K\left(\phi\left(\xi_{i}+\eta\right)\right), \quad i=1, \ldots, N .
$$

Next we define

$$
\begin{gathered}
V_{1}(x, t):=V\left(x+\delta_{1}, t ; \xi_{1}+\eta, k\right), \\
V_{i}(x, t):=V\left(x-c_{*} \sum_{j=1}^{i-1} T_{j}+\delta_{i}, t ; \xi_{i}+\eta, k\right), \quad t \in\left[0, T_{i}\right], \quad i=2, \ldots, N,
\end{gathered}
$$

where

$$
\begin{array}{rlrl}
T_{i} & :=T\left(\xi_{i}+\eta, k\right), & & i=1, \ldots, N, \\
\delta_{0} & :=\eta, & & \\
\delta_{i+1} & :=\delta_{i}+\xi_{i+1}-\xi_{i}-\left(k-c_{*}\right) T_{i} & i & i=1, \ldots, N-1 .
\end{array}
$$


The definition of $\delta_{i}$ leads to

$$
\begin{aligned}
0 & <\delta_{i+1}=\eta+\sum_{j=1}^{i}\left\{\xi_{i+1}-\left[\xi_{j}+\left(k-c_{*}\right) T_{j}\right]\right\} \\
& \leq \delta_{N}=N \eta+\sum_{j=1}^{N-1}\left\{\xi_{j+1}-\left[\xi_{j}+\eta+\left(k-c_{*}\right) T_{j}\right]\right\} .
\end{aligned}
$$

Then, applying Lemma 4.4,

$$
\lim _{\eta \rightarrow 0^{+}} \lim _{k \rightarrow c_{*}^{+}} \delta_{i}=0, \quad i=1, \ldots, N .
$$

From (4.13) and (4.14) it follows that

$$
\begin{aligned}
u_{0}^{R}(x) & \leq V_{1}(x, 0) & & \forall x \in \mathbb{R}, \\
V_{i}\left(x, T_{i}\right) & \leq V_{i+1}(x, 0) & & \forall x \in \mathbb{R}, \forall i=1, \ldots, N-1 .
\end{aligned}
$$

By the comparison principle (Theorem 4.2) applied to $u^{R}(x, t)$ and $V_{1}(x, t)$ we get the following:

$$
u^{R}(x, t) \leq V_{1}(x, t) \quad \text { a.e. in } \mathbb{R}, \forall t \in\left[0, T_{1}\right]
$$

Thus

$$
u^{R}\left(x, T_{1}\right) \leq V_{1}\left(x, T_{1}\right) \leq V_{2}(x, 0) .
$$

Now we use Theorem 4.2 once again to obtain

$$
u^{R}\left(x, T_{1}+t\right) \leq V_{2}(x, t) \quad \text { a.e. in } \mathbb{R}, \forall t \in\left[0, T_{2}\right]
$$

By applying $n$ times the comparison principle, we derive

$$
u^{R}\left(x, \sum_{j=1}^{n-1} T_{j}+t\right) \leq V_{n}(x, t) \quad \text { a.e. in } \mathbb{R}, \forall t \in\left[0, T_{n}\right] .
$$

Moreover, since $u_{0}^{R}(x) \geq \phi_{*}(x)$,

$$
\phi_{*}\left(x-c_{*} t\right) \leq u^{R}(x, t) \quad \text { a.e. in } \mathbb{R}, \forall t \in[0,+\infty) .
$$

Given $\varepsilon>0$, let us set

$$
N_{\varepsilon}:=\bigcup_{i=1}^{N}\left(\xi_{i}-\frac{\varepsilon}{4 N}, \xi_{i}+\frac{\varepsilon}{4 N}\right)
$$

then meas $N_{\varepsilon}=\varepsilon / 2$.

From formulas (4.15) and (4.16), we obtain

$$
\left|u^{R}\left(y+c_{*} t, t\right)-\phi_{*}(y)\right| \leq V_{n}\left(y+c_{*} t, t-\sum_{j=1}^{N-1} T_{j}\right)-\phi_{*}(y)
$$

for almost any $y \in \mathbb{R}$ and for any $t \in\left[\sum_{j=1}^{N-1} T_{j}, \sum_{j=1}^{N} T_{j}\right]$. 
From (4.14) we get

$$
V_{N}\left(y+c_{*} t, t-\sum_{j=1}^{N-1} T_{j}\right)-\phi_{*}(y)= \begin{cases}\phi_{*}\left(y+\delta_{N}\right)-\phi_{*}(y), & y<\left(k-c_{*}\right) t \\ 1-\phi_{*}(y), & y>\left(k-c_{*}\right) t\end{cases}
$$

Given $\theta>0$, we can choose $\eta=\eta(\theta)$ and $k=k(\theta)$ such that

$$
\sup _{\mathbb{R} \backslash N_{\varepsilon}}\left|\phi_{*}\left(y+\delta_{N}\right)-\phi_{*}(y)\right|<\theta .
$$

Moreover, since the function $1-\phi_{*}(y)$ decreases monotonically to zero as $y \rightarrow+\infty$, there is $t_{0}=t_{0}(\eta, k)$ such that

$$
1-\phi_{*}(y) \leq 1-\phi_{*}\left(\left(k-c_{*}\right) t\right)<\theta \quad \forall y>\left(k-c_{*}\right) t,
$$

for any $t>t_{0}$.

Coming back to (4.17), we deduce that

$$
\text { ess } \sup _{\mathbb{R} \backslash N_{\varepsilon}}\left|u^{R}\left(y+c_{*} t, t\right)-\phi_{*}(y)\right|<\theta,
$$

for any $t>t_{0}$.

If $T_{n}=+\infty$, passing to the limit as $t \rightarrow+\infty$, we get the conclusion. In the case $T_{n}<+\infty$ for any $k<c_{*}$, the same estimate follows from Lemma 4.4. In fact, it implies that

$$
\lim _{k \rightarrow c_{*}^{+}} y_{T_{N}^{\prime}}=+\infty \text {. }
$$

Thus, we can choose $k$ sufficiently close to $c_{*}$ in such a way that (4.18) holds. From (4.19) we get (2.7).

To prove $L^{p}$-convergence note that, by $(4.17)$,

$$
\begin{aligned}
\left\|u^{R}(\cdot, t)-\phi_{*}\left(\cdot-c_{*} t\right)\right\|_{L^{p}(\mathbb{R})}^{p} \leq \int_{-\infty}^{\left(k-c_{*}\right) t}\left|\phi_{*}\left(y+\delta_{n}\right)-\phi_{*}(y)\right|^{p} d y & +\int_{\left(k-c_{*}\right) t}^{+\infty}\left(1-\phi_{*}(y)\right)^{p} d y .
\end{aligned}
$$

It follows, by properties of $\phi_{*}$, that

$$
\int_{\mathbb{R}}\left|\phi_{*}(y+\delta)-\phi_{*}(y)\right|^{p} d y=\delta\left|\phi_{*}(+\infty)-\phi_{*}(-\infty)\right|^{p}=\delta .
$$

Therefore,

$$
\left\|u^{R}(\cdot, t)-\phi_{*}\left(\cdot-c_{*} t\right)\right\|_{L^{p}(\mathbb{R})}^{p} \leq \delta_{n}+\int_{\left(k-c_{*}\right) t}^{+\infty}\left(1-\phi_{*}(y)\right)^{p} d y .
$$

For any $\varepsilon>0$, choose $k$ such that $\delta_{n}<\varepsilon / 2$. Then there exists $t_{0}=t_{0}(\varepsilon, k)$ such that

$$
\int_{\left(k-c_{*}\right) t}^{+\infty}\left(1-\phi_{*}(y)\right)^{p} d y<\frac{\varepsilon}{2} \text {. }
$$

Therefore,

$$
\left\|u^{R}(\cdot)-\phi_{*}\left(\cdot-c_{*} t\right)\right\|_{L^{p}(\mathbb{R})}^{p}<\varepsilon \quad \forall t>t_{0} .
$$

The proof is complete. 
Next we prove Theorem 2.5.

Proof of Theorem 2.5. By assumptions, $c_{*}=0$ and $f^{\prime}$ is strictly increasing in $\left[0, a_{1}\right]$. Then there exists $\varepsilon_{0} \in\left(0, a_{1}\right)$ such that for any $\varepsilon \in\left(0, \varepsilon_{0}\right)$, there is $h_{\varepsilon} \in\left(0, f^{\prime}(\varepsilon)\right)$ such that

$$
f(s)>f(\varepsilon)+h_{\varepsilon}(s-\varepsilon) \quad \forall s>\varepsilon .
$$

In fact, suppose by contradiction that for any $\delta>0$ there exists $\varepsilon \in(0, \delta)$ such that for any $h \in\left(0, f^{\prime}(\varepsilon)\right)$ there is $s_{h, \varepsilon}$ such that

$$
f\left(s_{h, \varepsilon}\right)=f(\varepsilon)+h\left(s_{h, \varepsilon}-\varepsilon\right) .
$$

Let $H(s):=f(s)-f(\varepsilon)-h(s-\varepsilon)$. Then $H(\varepsilon)=0, H^{\prime}(\varepsilon)>0, H^{\prime \prime}(\varepsilon)>0$. Hence $H(s)>0$ for any $s \in\left(\varepsilon, a_{1}\right]$ and $s_{h, \varepsilon}>a_{1}$.

Let $h_{n}$ be a sequence converging to zero as $n \rightarrow+\infty$, and let $s_{n}:=s_{h_{n}, \varepsilon}$. Then (taking a subsequence if needed) $s_{n}$ converges to some $s_{\varepsilon} \geq a_{1}$ as $n \rightarrow+\infty$. For such an $s_{\varepsilon}$ we deduce, from (4.21), $f\left(s_{\varepsilon}\right)=f(\varepsilon)$, with $\varepsilon \in(0, \delta), s_{\varepsilon} \geq a_{1}$.

Letting $\delta \rightarrow 0$, we deduce that there exists $s_{0} \geq a_{1}$ such that $f\left(s_{0}\right)=f(0)$, which contradicts the hypothesis. Therefore (4.20) holds.

Now, given $\varepsilon \in\left(0, \varepsilon_{0}\right)$, we define

$$
\int_{\varepsilon}^{\phi_{\varepsilon}(x)} \frac{f^{\prime}(s)}{g(s)} d s=x,
$$

and

$$
V_{\varepsilon}(x, t)= \begin{cases}\phi_{\varepsilon}(x), & x<h_{\varepsilon} t, \\ 1, & x>h_{\varepsilon} t .\end{cases}
$$

Since (4.20) holds, the function $V_{\varepsilon}$ proves to be an entropy supersolution of problem (2.1) with $u_{0} \leq u_{0}^{R}$ for any $\varepsilon \in\left(0, \varepsilon_{0}\right)$.

Then, for any $M \in \mathbb{R}$,

$$
\begin{aligned}
\|u(\cdot, t)\|_{L^{\infty}(-\infty, M)} & \leq\left\|u^{R}(\cdot, t)\right\|_{L^{\infty}(-\infty, M)} \\
& \leq\left\|V_{\varepsilon}(\cdot, t)\right\|_{L^{\infty}(-\infty, M)}=\phi_{\varepsilon}(M) \quad \forall t>\frac{M}{h_{\varepsilon}} .
\end{aligned}
$$

Since $f^{\prime} / g$ is not integrable at 0 , we deduce that

$$
\lim _{\varepsilon \rightarrow 0} \phi_{\varepsilon}(M)=0 \text {. }
$$

Thus, for any $\varepsilon>0$, there is $T_{\varepsilon}=M / h_{\varepsilon}$ such that

$$
\|u(\cdot, t)\|_{L^{\infty}(-\infty, M)}>\varepsilon \quad \forall t>T_{\varepsilon} .
$$

This completes the proof.

\section{REFERENCES}

[1] C. M. Dafermos, Characteristics in hyperbolic conservation laws. A study of the structure and the asymptotic behavior of solutions, in Nonlinear Analysis and Mechanics, Vol. 1 (R. J. Knops, ed.), Research Notes in Mathematics 17, Pitman, 1977, pp. 1-58

[2] C. M. Dafermos, Generalized characteristics and the structure of solutions of hyperbolic conservation laws, Indiana Univ. Math. 26, 1097-1119 (1977) 
[3] C. M. Dafermos, Large time behavior of solutions of hyperbolic balance laws, Bull. Soc. Math. Grèce (N.S.) 25, 15-29 (1984)

[4] C. M. Dafermos, Regularity and large time behaviour of solutions of a conservation law without convexity, Proc. Roy. Soc. Edinburgh Sect. A 99, 201-239 (1985)

[5] H. Fan and J. K. Hale, Attractors in inhomogeneous conservation laws and parabolic regularizations, Trans. Amer. Math. Soc. 347, 1239-1254 (1995)

[6] H. Fan and J. K. Hale, Large-time behavior in inhomogeneous conservation laws, Arch. Rational Mech. Anal. 125, 201-216 (1993)

[7] B. T. Hayes, Stability of solutions to a destabilized Hopf equation, Comm. Pure Appl. Math. 48, 157-166 (1995)

[8] A. Kolmogorov, I. Petrovsky, and N. Piscounov, Etude de l'équation de la diffusion avec croissance de la quantité de matière et son application à un problème biologique, Boll. Univ. Moscow Ser. Internat. Sec. A 1, 1-25 (1937)

[9] S. N. Kružkov, First order quasilinear equations in several independent variables, Mat. Sb. 51, 99-128 (1960) (Russian); English transl. in Amer. Math. Soc. Transl. (2) 42, 199-231 (1964)

[10] S. N. Kružkov and N. S. Petrosjan, Asymptotic behaviour of the solutions of the Cauchy problem for nonlinear first order equations, Uspekhi Mat. Nauk 42, 3-40 (1987) (Russian); English transl. in Russian Math. Surveys 42, 1-47 (1987)

[11] P. D. Lax, Hyperbolic systems of conservation laws II, Comm. Pure Appl. Math. 10, 537-566 (1957)

[12] T. P. Liu, Invariants and asymptotic behavior of solutions of a conservation law, Proc. Amer. Math. Soc. 71, 227-231 (1978)

[13] A. N. Lyberopoulos, A Poincaré-Bendixson theorem for scalar balance laws, Proc. Roy Soc. Edinburgh Sect. A 124, 589-607 (1994)

[14] A. N. Lyberopoulos, Asymptotic oscillations of solutions of scalar conservation laws with convexity under the action of a linear excitation, Quart. Appl. Math. 48, 755-765 (1990)

[15] A. N. Lyberopoulos, Large time structure of solutions of scalar conservation laws without convexity in the presence of a linear source field, J. Differential Equations 99, 342-380 (1992)

[16] C. Mascia, Continuity in finite time of entropy solutions for nonconvex conservation laws with reaction term, Comm. Partial Differential Equations 23, 913-931 (1998)

[17] C. Mascia, Travelling wave solutions for a balance law, Proc. Roy. Soc. Edinburgh Sect. A 127, 567-593 (1997)

[18] C. Mascia and C. Sinestrari, The perturbed Riemann problem for a balance law, Advances in Differential Equations 2, 779-810 (1997)

[19] R. Natalini and A. Tesei, On a class of perturbed conservation laws, Adv. in Appl. Math. 13, 429-453 (1992)

[20] O. A. Oleinik, Uniqueness and stability of the generalized solution of the Cauchy problem for a quasilinear equation, Amer. Math. Soc. Transl. Ser. 2 33, 285-290 (1964)

[21] C. Sinestrari, Asymptotic profile of solutions of conservation laws with source, Differential Integral Equations 9, 499-525 (1966)

[22] C. Sinestrari, Large time behaviour of solutions of balance laws with periodic initial data, NoDEA Nonlinear Differential Equations Appl. 2, 111-131 (1995)

[23] C. Sinestrari, The Riemann problem for an inhomogeneous conservation law without convexity, SIAM J. Math. Anal. 28, 109-135 (1997)

[24] C. Sinestrari, Instability of discontinuous traveling waves for hyperbolic balance laws, J. Differential Equations 134, 269-285 (1997)

[25] J. Smoller, Shock waves and reaction-diffusion equations, Springer-Verlag, New York, 1983

[26] A. I. Volpert, V. A. Volpert, and V. A. Volpert, Traveling wave solutions of parabolic systems, Translations of Mathematical Monographs 140, American Mathematical Society, Providence, RI, 1994

[27] H. F. Weinberger, Long-time behavior for a regularized scalar conservation law in the absence of genuine nonlinearity, Ann. Inst. H. Poincaré (Anal. Non Linéaire) 7, 407-425 (1990) 